\title{
Modeling the effectiveness of shielding in the earth-moon-mars radiation environment using PREDICCS: five solar events in 2012
}

\author{
Philip R. Quinn ${ }^{1, *}$, Nathan A. Schwadron ${ }^{1}$, Larry W. Townsend ${ }^{2}$, Robert F. Wimmer-Schweingruber ${ }^{3}$, \\ Anthony W. Case ${ }^{4}$, Harlan E. Spence ${ }^{1}$, Jody K. Wilson ${ }^{1}$, and Colin J. Joyce ${ }^{1}$ \\ 1 Space Science Center, University of New Hampshire, Durham, NH 03824, USA \\ *Corresponding author: prm29@wildcats.unh.edu \\ 2 Department of Nuclear Engineering, University of Tennessee, Knoxville, TN 37996, USA \\ 3 Institute for Experimental and Applied Physics, Christian-Albrechts-University of Kiel, Kiel 24118, Germany \\ 4 High Energy Astrophysics Division, Harvard Smithsonian Center for Astrophysics, Cambridge, MA 02138, USA
}

Received 27 October 2016 / Accepted 9 June 2017

\begin{abstract}
Radiation in the form of solar energetic particles (SEPs) presents a severe risk to the short-term health of astronauts and the success of human exploration missions beyond Earth's protective shielding. Modeling how shielding mitigates the dose accumulated by astronauts is an essential step toward reducing these risks. PREDICCS (Predictions of radiation from REleASE, EMMREM, and Data Incorporating the CRaTER, COSTEP, and other SEP measurements) is an online tool for the near real-time prediction of radiation exposure at Earth, the Moon, and Mars behind various levels of shielding. We compare shielded dose rates from PREDICCS with dose rates from the Cosmic Ray Telescope for the Effects of Radiation (CRaTER) onboard the Lunar Reconnaissance Orbiter (LRO) at the Moon and from the Radiation Assessment Detector (RAD) on the Mars Science Laboratory (MSL) during its cruise phase to Mars for five solar events in 2012 when Earth, MSL, and Mars were magnetically well connected. Calculations of the accumulated dose demonstrate a reasonable agreement between PREDICCS and RAD ranging from as little as $2 \%$ difference to $54 \%$. We determine mathematical relationships between shielding levels and accumulated dose. Lastly, the gradient of accumulated dose between Earth and Mars shows that for the largest of the five solar events, lunar missions require aluminum shielding between $1.0 \mathrm{~g} \mathrm{~cm}^{-2}$ and $5.0 \mathrm{~g} \mathrm{~cm}^{-2}$ to prevent radiation exposure from exceeding the 30-day limits for lens and skin. The limits were not exceeded near Mars.
\end{abstract}

Key words. Mars - Moon - Radiation environment - Human exploration - Exposure

\section{Introduction}

Human space exploration beyond the protective shielding of Earth's atmosphere and magnetosphere is hindered due to intense radiation that threatens the short-term and long-term health of astronauts (Cucinotta et al. 2010). In the EarthMoon-Mars radiation environment, the two primary sources of energetic particles are galactic cosmic rays (GCRs) and solar energetic particles (SEPs).

Galactic cosmic rays (GCRs) are an enduring source of highly energetic particles with origins outside the heliosphere. GCRs typically originate from supernova explosions within the galaxy, diffuse into the heliosphere, and lead to a modest but persistent background dose rate in the Earth-Moon-Mars radiation environment. The intensity of the GCR background varies with solar activity - becoming largest near solar minimum and smallest near solar maximum. For average solar condition, the long-term exposure of GCRs can limit female and male astronauts' careers to 900 and 1200 days, respectively, due to the risk of cancer induction and mortality (Cucinotta et al. 2015).

Solar energetic particles (SEPs) are produced from solar events such as solar flares and coronal mass ejections (CMEs). Solar events are sporadic and short-lived, resulting in an increase of high-energy particle flux by many orders of magnitude over the span of hours to days. Astronauts exposed to radiation dose above $50 \mathrm{cGy}$ from SEPs can experience Acute Radiation Syndrome (ARS) (Anno et al. 1989) including prodromal effects (e.g. nausea, vomiting, anorexia, fatigue), skin injury, the depletion of blood-forming organs (BFOs), and death (Wu et al. 2009; Cucinotta et al. 2010). The NASA 30-day Permissible Exposure Limits (PELs) for lens, skin, BFOs, heart, and central nervous system (CNS) have been implemented for the prevention of these health risks. These limits are given in Table 1 (NAS/NRC 1967, 1970; NCRP 1989, 2000).

To prevent health risks to astronauts caused by exposure to SEPs, it is imperative that the exposure be mitigated by the shielding of their spacesuit, spacecraft, or shelter. Modeling plays an important role in predicting the necessary level of shielding. The Earth-Moon-Mars Radiation Environment Module (EMMREM) (Schwadron et al. 2010) has been used to find the radial gradient of shielded dose rates with comparisons to Ulysses for the Halloween 2003 solar event (Kozarev et al. 2010) and May 2003 solar event (Dayeh et al. 2010). Predictions of radiation from REleASE, EMMREM, and Data Incorporating the CRaTER, COSTEP, and other SEP measurements (PREDICCS) (http://prediccs.sr.unh.edu) is an online tool for the near real-time prediction of radiation exposure at Earth, the Moon, and Mars behind various levels of shielding. 
Table 1. NASA 30-day permissible exposure limits.

\begin{tabular}{lc}
\hline \hline Organ & 30 -Day limit \\
\hline Lens & 100 cGy-eq \\
Skin & 150 cGy-eq \\
BFO & 25 cGy-eq \\
Heart & 25 cGy-eq \\
CNS & 50 cGy-eq \\
\hline
\end{tabular}

Notes. See Section 4.2 for explanation on dose units.

PREDICCS has been verified to provide a credible characterization of the lunar radiation environment (Schwadron et al. 2012; Joyce et al. 2013).

In this paper, we use PREDICCS to predict the level of shielding required for human missions in the Earth-MoonMars radiation environment such that astronauts' exposure to radiation does not exceed the 30-day dose limits. SEP measurements from five solar events in 2012 are used by PREDICCS to simulate the transport of SEPs across interplanetary space and through an Aluminum slab shield and $\mathrm{H}_{2} \mathrm{O}$ slab target. We consider Aluminum slab shields of $0.3 \mathrm{~g} \mathrm{~cm}^{-2}$, $1.0 \mathrm{~g} \mathrm{~cm}^{-2}, 5.0 \mathrm{~g} \mathrm{~cm}^{-2}$, and $10.0 \mathrm{~g} \mathrm{~cm}^{-2}$ (proxies for thin spacesuit, nominal spacecraft, thick spacecraft, and stormshelter shielding, respectively) and $\mathrm{H}_{2} \mathrm{O}$ slab targets of $1.0 \mathrm{~g} \mathrm{~cm}^{-2}$ $\mathrm{H}_{2} \mathrm{O}$ (proxy for lens and skin) and $10.0 \mathrm{~g} \mathrm{~cm}^{-2} \mathrm{H}_{2} \mathrm{O}$ (proxy for BFOs, heart, and CNS). During the five solar events in 2012, the Radiation Assessment Detector (RAD) (Hassler et al. 2012) on the Mars Science Laboratory (MSL) (Grotzinger et al. 2012) measured dose rates during its cruise phase from Earth to Mars. MSL was magnetically well connected to Earth early in the cruise phase and to Mars later in the cruise phase (Posner et al. 2013) - allowing for good comparison to PREDICCS for interplanetary space between Earth and Mars. We additionally compare dose rates to the Cosmic Ray Telescope for the Effects of Radiation (CRaTER) (Spence et al. 2010) onboard the Lunar Reconnaissance Orbiter (LRO) (Chin et al. 2007) orbiting the Moon.

\section{PREDICCS, CRaTER, and RAD overview}

\subsection{PREDICCS}

PREDICCS is an online system used to predict and forecast the interplanetary radiation environment. The consituents of PREDICCS are described below.

First, PREDICCS uses near real-time SEP proton measurements from various instruments depending on the years of operation. For the five 2012 solar events of this study, PREDICCS used proton measurements from the suite of instruments called EPS/HEPAD (Energetic Particle Sensors/High Energy Proton and Alpha Detector) onboard Geostationary Operational Environmental Satellite-13 (GOES-13). EPS measures protons in seven energy channels ranging from $0.7 \mathrm{MeV}$ to $900 \mathrm{MeV}$. HEPAD measures protons in four energy channels ranging from $330 \mathrm{MeV}$ to over $700 \mathrm{MeV}$. Uncertainty in measurements for all energy channels is estimated to be about 25\% (J. Rodriguez, personal communication). The time-series SEP proton intensities are shown in Figure 1. The starting and ending times are arbitrarily taken to encompass the events.

Second, the time-series SEP proton measurements are used as input for the Energetic Particle Radiation Environment
Module (EPREM) (Schwadron et al. 2010; Quinn et al. 2016). EPREM simulates particle transport across interplanetary space. This is done using a Lagrangian scheme with nodes that spawn on a rotating inner boundary. The nodes are then carried out with the solar wind. At each time-step, the focused transport equation (Skilling 1971; Ruffolo 1995) and convection-diffusion equation (Jokipii \& Levy 1977; Lee \& Fisk 1981 ) are solved along each magnetic field line (a connected list of nodes). EPREM therefore takes into account convection, streaming, adiabatic focusing, adiabatic cooling, pitch-angle scattering, perpendicular diffusion, and particle drift. For PREDICCS, EPREM outputs proton flux at Earth and Mars. Third, proton flux from EPREM is used as input for the BaRYoN TRaNsport (BRYNTRN) model (Wilson et al. 1989). BRYNTRN simulates the transport of the SEP protons and their secondary particles through an Aluminum slab shield and $\mathrm{H}_{2} \mathrm{O}$ slab target. In PREDICCS, we consider Aluminum slab shields of $0.3 \mathrm{~g} \mathrm{~cm}^{-2}, 1.0 \mathrm{~g} \mathrm{~cm}^{-2}, 5.0 \mathrm{~g} \mathrm{~cm}^{-2}$, and $10.0 \mathrm{~g} \mathrm{~cm}^{-2}$ (proxies for thin spacesuit, nominal spacecraft, thick spacecraft, and stormshelter shielding, respectively) and $\mathrm{H}_{2} \mathrm{O}$ slab targets of $1.0 \mathrm{~g} \mathrm{~cm}^{-2} \mathrm{H}_{2} \mathrm{O}$ (proxy for lens and skin) and $10.0 \mathrm{~g} \mathrm{~cm}^{-2} \mathrm{H}_{2} \mathrm{O}$ (proxy for BFOs, heart, and CNS).

\subsection{CRaTER}

The CRaTER instrument onboard LRO characterizes the lunar radiation environment. Silicon solid-state detectors measure the effects of ionizing energy loss in matter due to SEPs and GCRs. The combined dose from the first pair of thin and thick zenith-facing silicon detectors (D1-D2) is an excellent proxy for lens exposure behind $0.22 \mathrm{~g} \mathrm{~cm}^{-2}$ aluminum shielding.

We combine the hourly averaged dose rates detected by the D1-D2 detectors. Since the LET coverage of the two detectors overlaps, we limit the energy range of each detector to prevent the possibility of double-counting dose contributions (Schwadron et al. 2012).

To compare to PREDICCS, the dose measured in CRaTER's D1-D2 silicon detectors must be converted to dose in water. The dose is calculated by summing over all the energy depositions in the detectors and dividing by the mass of silicon. The lower ionization potential of water compared to silicon results in larger energy deposition per unit mass for a particle with a given charge and velocity. Schwadron et al. (2012) determined the conversion between dose in CRaTER's D1-D2 silicon detectors and dose in water to be a factor of 1.33 .

The measurements of CRaTER are made at an altitude above the lunar surface where less than half the sky is blocked by the Moon. To adjust the measurements to the surface of the Moon where half the sky is blocked, the dose rates are multiplied by (Schwadron et al. 2012)

$$
F=\frac{1}{1+\sqrt{1-\left[R_{\mathrm{M}} /\left(R_{\mathrm{M}}+h\right)\right]^{2}}},
$$

where $h$ is the altitude of CRaTER above the lunar surface and $R_{\mathrm{M}}$ is the radius of the Moon.

The CRaTER detection electronics are sufficiently fast to pulse shape (i.e., energy) analyze approximately $90 \%$ of energetic particles incident on the detectors during the most extreme solar events (Spence et al. 2010). To account for the remaining $10 \%$ of incident particles not energy analyzed during high flux intervals, the CRaTER electronics has a feature that counts the total number of particle events per unit 
(a)

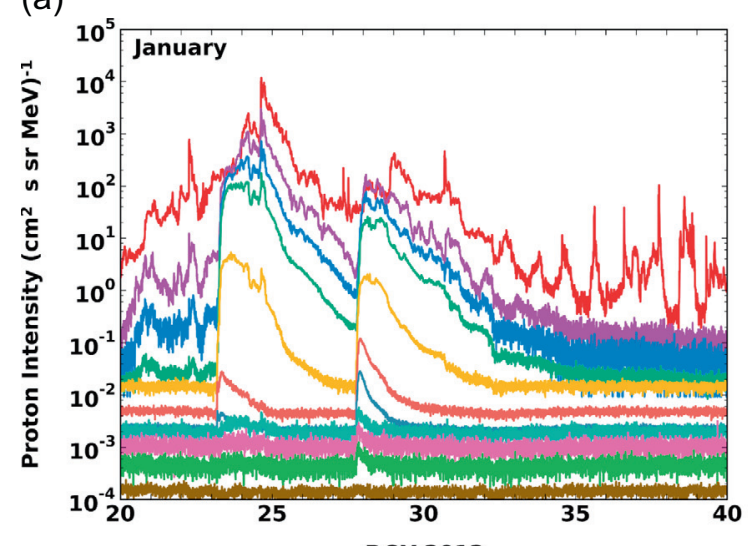

(c)

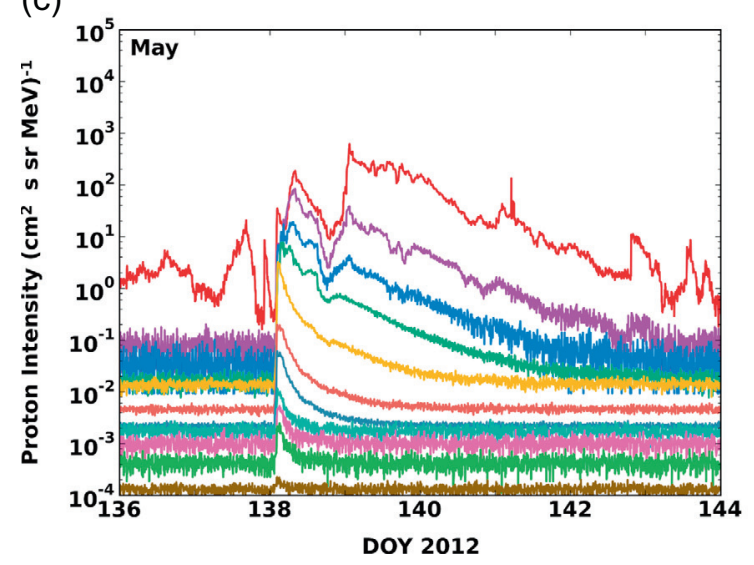

(b)

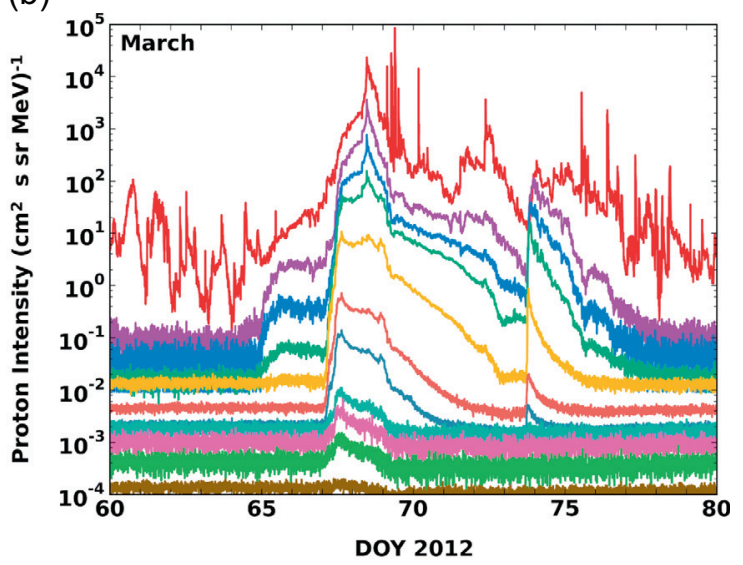

DOY 2012

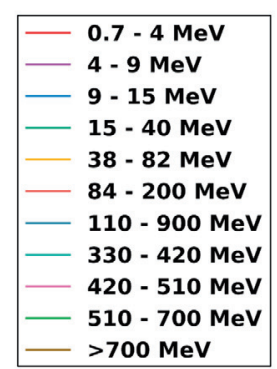

Fig. 1. Five-min averages of proton intensities measured by GOES-13/EPS/HEPAD during the January, March, and May 2012 solar events.

time that pass the detection threshold, including particles that were energy analyzed and those that were not. By multiplying the dose rate by the ratio of total particle events counted to particle events analyzed, we effectively compensate for the rate limit. This approach makes the reasonable assumption that the spectral properties of the $10 \%$ of particles not analyzed match the $90 \%$ of particles that were analyzed.

CRaTER also contains a microdosimeter chip (Mazur et al. 2011). The microdosimeter includes a $25 \mathrm{~mm}^{2}$ silicon detector used to measure the total ionizing dose. Due to being inside the housing of CRaTER, one side of the microdosimeter views the lunar surface through $\sim 0.89 \mathrm{~g} \mathrm{~cm}^{-2}$ aluminum shielding while the other side of the microdosimeter views deep space through $\sim 2.28 \mathrm{~g} \mathrm{~cm}^{-2}$ aluminum shielding (Mazur et al. 2011).

The LRO spacecraft may provide a small addition to the shielding of CRaTER D1-D2 and possibly the microdosimeter. Looper et al. (2013) used Geant4 to simulate the CRaTER telescope and incoming particles. Part of the study included the electronics box and a simplified mass model of the entire LRO spacecraft. However, only a small fractional increase in protons due to the breaking of alphas in the spacecraft was found.

\subsection{RAD}

The RAD instrument onboard the Curiosity rover measures radiation dose in two detectors. The silicon detector (B) is mainly sensitive to charged particles while the plastic detector (E) is mainly sensitive to neutrons. Since SEP events are dominated by energetic protons, we use detector B for this

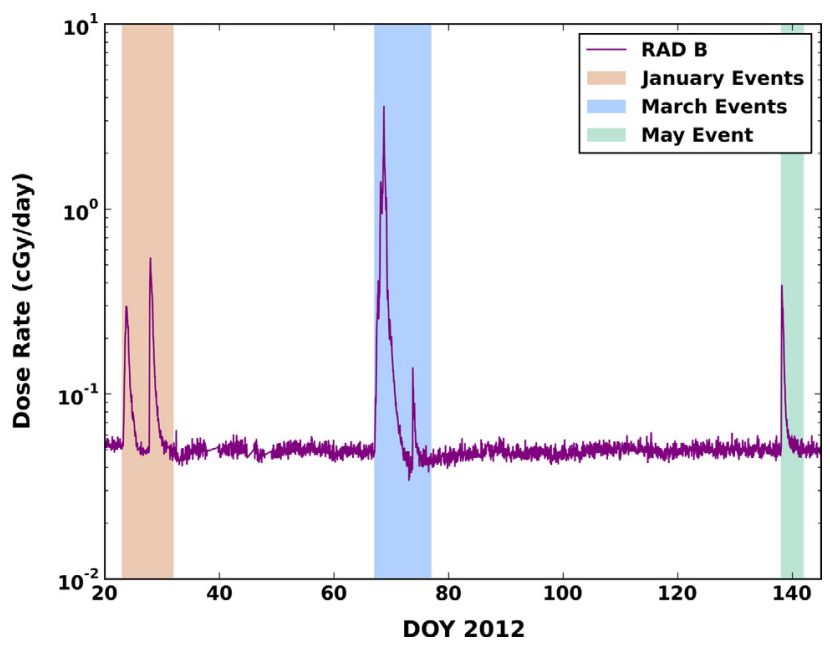

Fig. 2. Dose rate measured by the $B$ detector of the RAD instrument during its cruise to Mars in 2012. The dose rates have been converted from silicon to water. The five events observed by RAD are highlighted.

study. Similar to the two CRaTER detectors, the RAD dose rate is multiplied by 1.45 to convert dose in silicon to dose in water (Zeitlin et al. 2013).

Neutrons and gamma rays emitted from MSL's power supply were measured by RAD's B detector during cruise. Also measured were electrons and positrons produced by pair production of the more energetic gamma rays. Tests performed prior to launch determined the background dose rate 
(a)

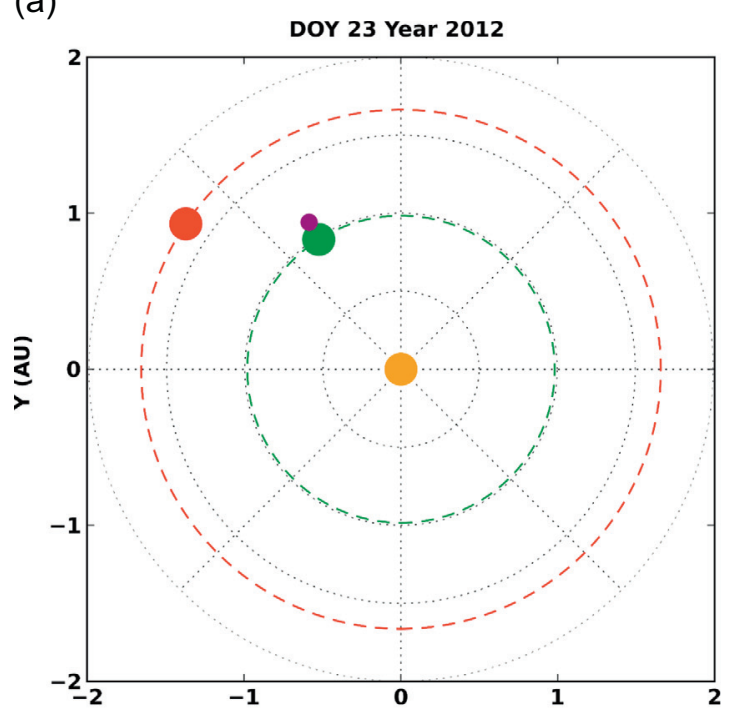

(b)

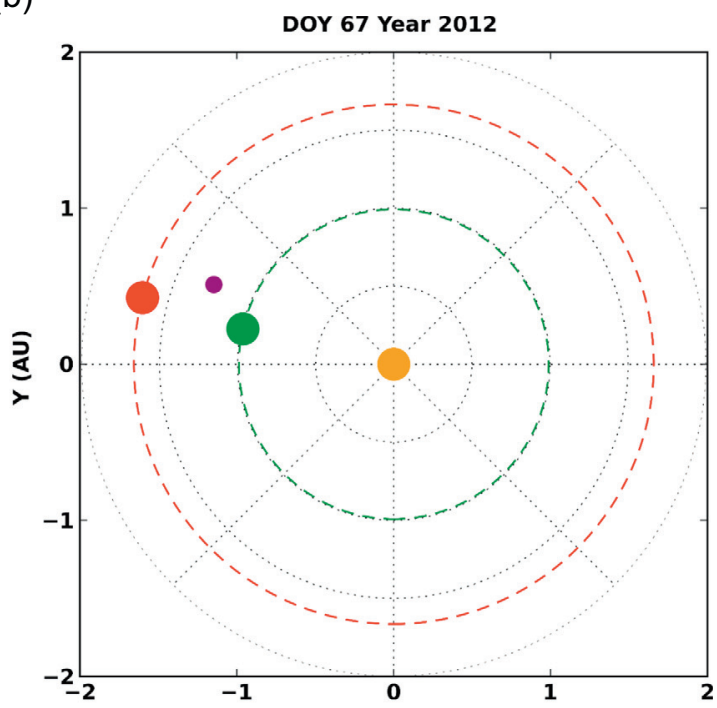

(c)

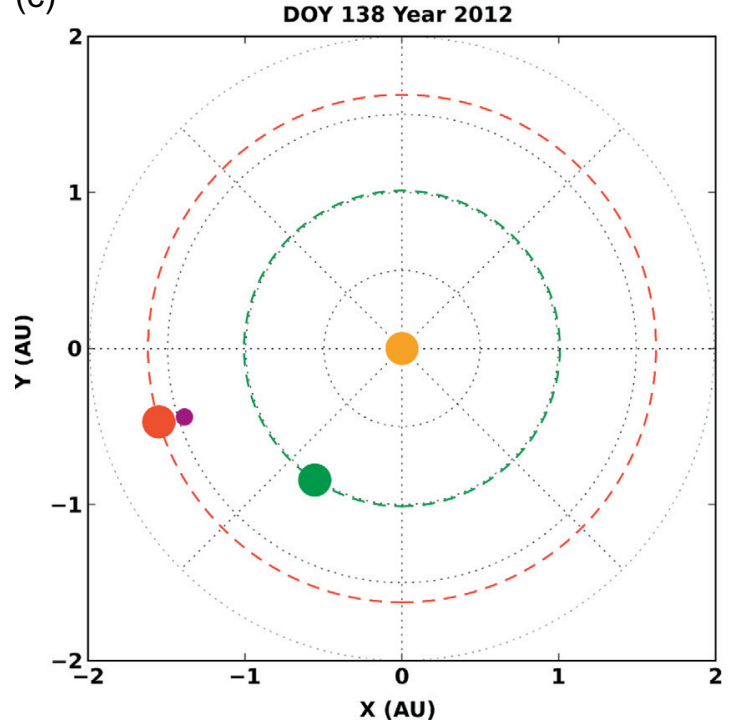

Fig. 3. Position in solar ecliptic coordinates of Earth (green), MSL (purple), and Mars (red) during the January (a), March (b), and May (c) 2012 solar events. The sun (orange) is shown at the center.

measured by RAD's B detector due to MSL's power supply to be approximately $0.0085 \mathrm{cGy} /$ day (Zeitlin et al. 2013). We subtract this background dose rate from all RAD measurements.

The spacecraft housing the Curiosity rover (and RAD) provided very inhomogeneous shielding from the radiation environment during MSL's cruise to Mars. About 30\% of incoming particles trajectories above RAD experienced aluminumequivalent shielding less than $5 \mathrm{~g} \mathrm{~cm}^{-2}$, about $50 \%$ below were shielded by $8-10 \mathrm{~g} \mathrm{~cm}^{-2}$, and the remaining shielded by more than $20 \mathrm{~g} \mathrm{~cm}^{-2}$. The average shielding surrounding RAD was then roughly $16 \mathrm{~g} \mathrm{~cm}^{-2}$ (Zeitlin et al. 2013).

\section{Solar event selection}

During MSL's cruise phase from Earth to Mars, the RAD instrument measured radiation dose rates from 6 December 2011 to 14 July 2012. Shown in Figure 2 are the five solar events observed by RAD during this time - two peaks in January, two in March, and one in May.

The January 2012 solar events consisted of a CME and long-duration M8.7 flare occurring on January 23 followed by a CME and a long-duration X1 flare occurring on January 27. The first CME originated from $\mathrm{N} 29^{\circ} \mathrm{W} 20^{\circ}$ and reached a peak speed of about $1600 \mathrm{~km} \mathrm{~s}^{-1}$ (Liu et al. 2013). The second $\mathrm{CME}$ originated from $\mathrm{N} 27^{\circ} \mathrm{W} 71^{\circ}$ and reached a peak speed of over $2600 \mathrm{~km} \mathrm{~s}^{-1}$ (Gopalswamy et al. 2015). The two events resulted in approximately 10 days of increased SEP flux observed at 1 AU. Figure 3a shows the locations of Earth, MSL, and Mars during the date of the first peak. The MSL spacecraft on its cruise to Mars was located at approximately $1.12 \mathrm{AU}$.

The March 2012 solar events were composed of a series of CMEs and flares occurring on March 5, March 7, March 9, March 10, and March 13 (Liu et al. 2013). The largest and most energetic was the X5.4 flare and CME occurring on March 7. The $\mathrm{CME}$ originated from $\mathrm{N} 17^{\circ} \mathrm{E} 21^{\circ}$ and 
(a)

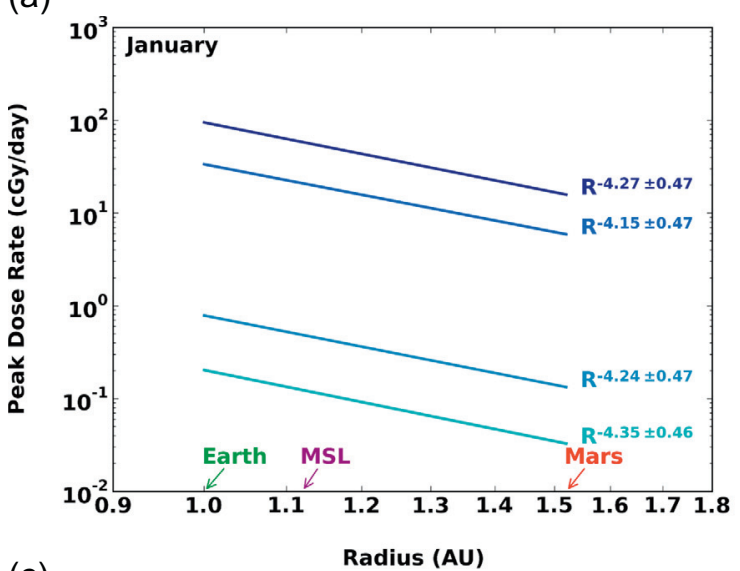

(c)

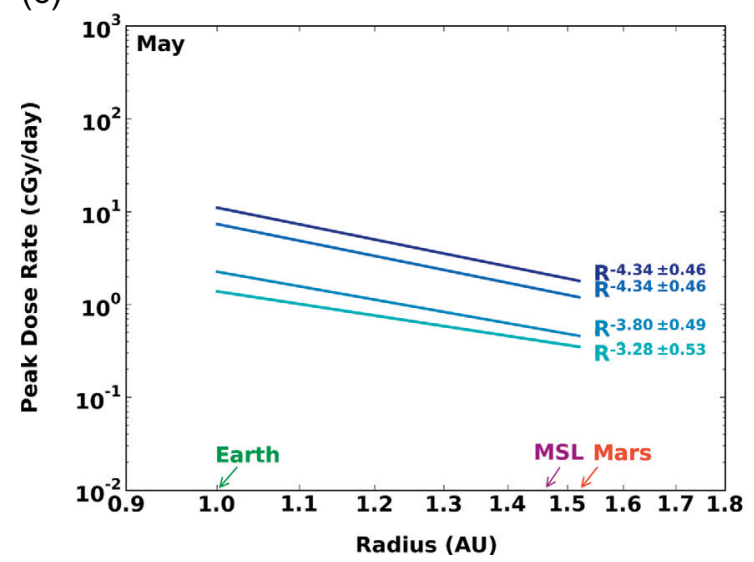

(b)

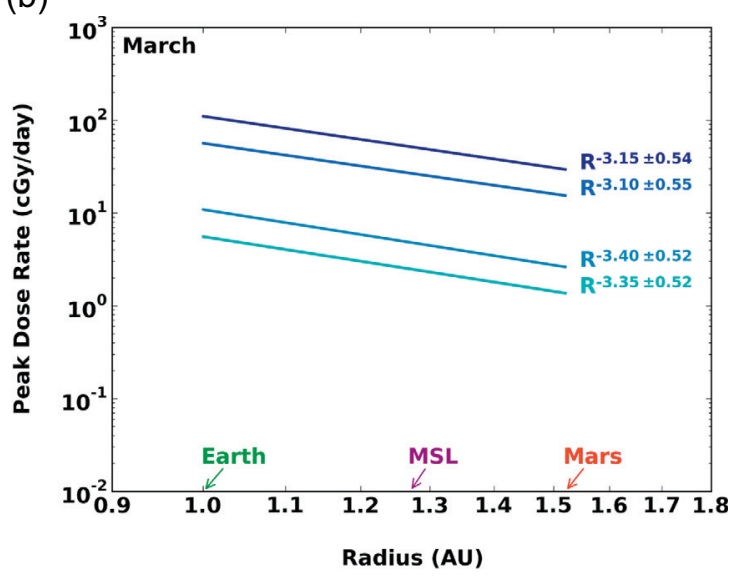

Fig. 4. Radial gradient of peak dose rate between Earth and Mars (see text). Dose rates are behind $0.3 \mathrm{~g} \mathrm{~cm}^{-2}, 1.0 \mathrm{~g} \mathrm{~cm}^{-2}, 5.0 \mathrm{~g} \mathrm{~cm}^{-2}$, and $10.0 \mathrm{~g} \mathrm{~cm}^{-2}$ aluminum shielding. In the bottom are the radial locations of Earth (green), MSL (purple), and Mars (red).

reached a peak speed of over $2000 \mathrm{~km} \mathrm{~s}^{-1}$. An additional CME of about $1500 \mathrm{~km} \mathrm{~s}^{-1}$ and X1.3 flare occurred nearly an hour later. SEP fluxes at $1 \mathrm{AU}$ increased for about 10 days from March 7 to March 17. MSL was located at approximately $1.27 \mathrm{AU}$.

The May 2012 solar event was made up of a long-duration M5.1 flare and CME with a peak speed of $1582 \mathrm{~km} \mathrm{~s}^{-1}$ (Li et al. 2013; Ding et al. 2016). SEP fluxes increased at $1 \mathrm{AU}$ for about 4 days from May 17 to May 21. During this solar event, MSL was at approximately 1.46 AU.

MSL remained well connected magnetically to Earth in the early phase of transit and to Mars in the later phase of transit (Posner et al. 2013). This allows for an accurate comparison between PREDICCS dose rate at Earth and Mars with the dose rate measured by MSL/RAD during the five solar events.

\section{Dose rates and accumulated dose}

In this section, we scale PREDICCS SEP dose rates to the location of MSL during each event, discuss dose, and calculate the accumulated dose. Comparisons are made to the 30-day lens and skin limit to assess the risk the solar events pose to the health of astronauts. All shielded dose rates simulated by PREDICCS use a $1.0 \mathrm{~g} \mathrm{~cm}^{-2} \mathrm{H}_{2} \mathrm{O}$ slab target unless specified otherwise.

\subsection{Scaling dose rates to MSL location}

PREDICCS produces shielded dose rates exclusively at the locations of Earth and Mars. These dose rates must be scaled from Earth during the earlier phase of MSL's trajectory and Mars during the later phase. Kozarev et al. (2010) and Dayeh et al. (2010) used EMMREM to simulate SEP transport during the Halloween 2003 and May 2003 solar events, respectively. The studies concluded that the peak dose rate as SEPs are transported along the interplanetary magnetic field - follows a power law when expressed as a function of heliocentric radius. Following these studies, we find the peak dose rate at Earth and Mars for each solar event and each level of shielding produced by PREDICCS. The shielded peak dose rates as a function of radius are shown in Figure 4 for the January event (Fig. 4a), March event (Fig. 4b), and May event (Fig. 4c). The location of Earth, MSL, and Mars is indicated at the bottom of each plot. The power law indices are displayed at the right of each fit. The indices of the January and May events are comparable to those of Kozarev et al. (2010). The indices of the March event are smaller than those of the January and May events, meaning we see a smaller ratio of dose rate between $1 \mathrm{AU}$ and Mars. One reason for this may be the higher speed of the March $7 \mathrm{CME}$. Another reason may be due to the CMEs that occurred prior, possibly disturbing the magnetic field and allowing for easier particle propagation for the March 7 CME. 
(a)

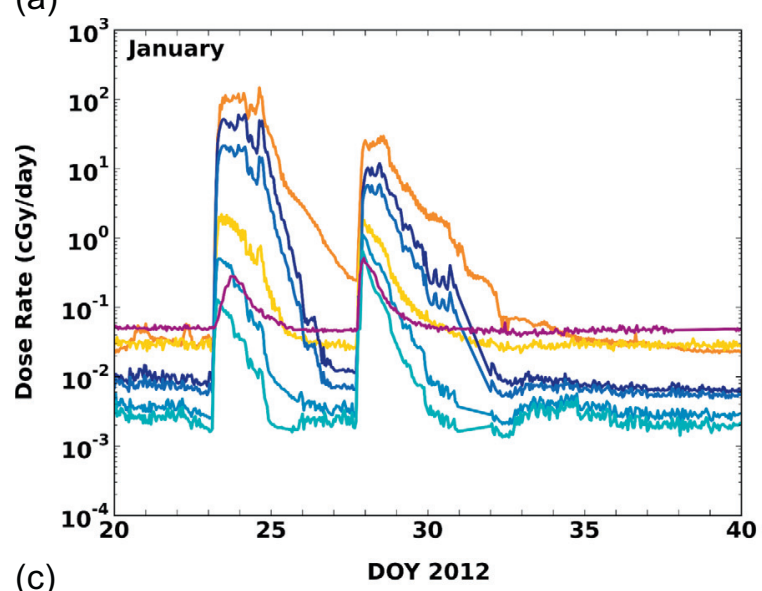

(c)

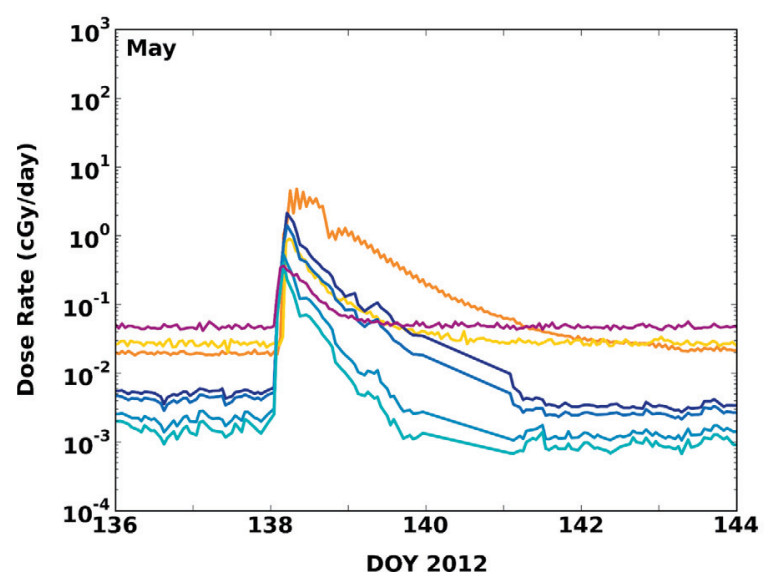

(b)
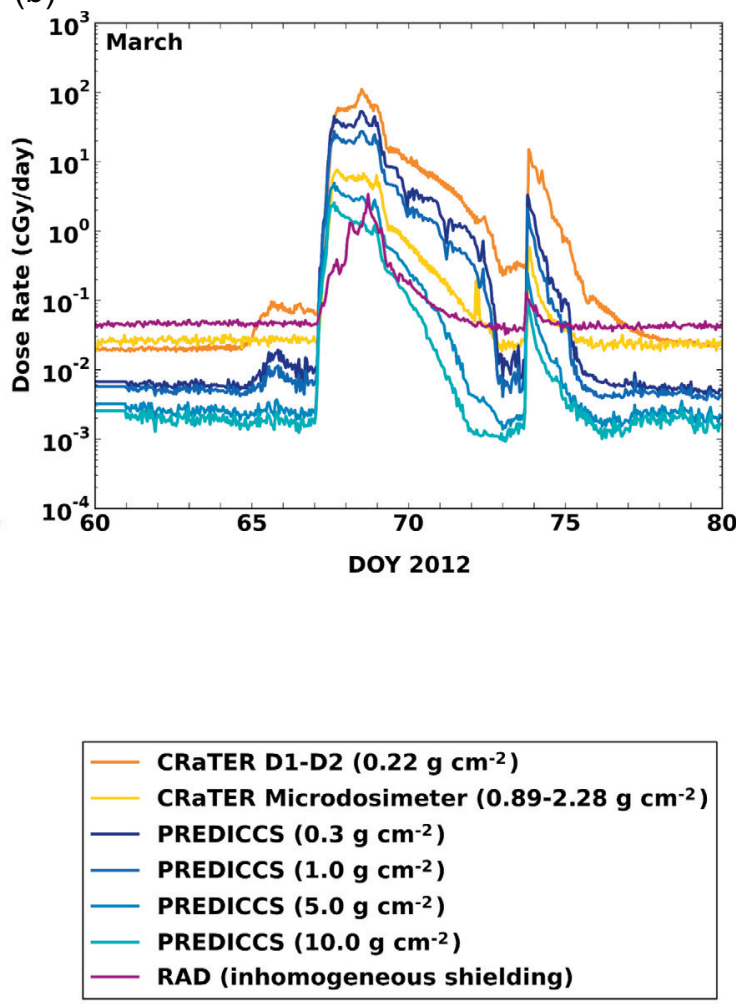

Fig. 5. Hourly averaged dose rates for the January event (a), March event (b), and May event (c). The beginning and ending times are arbitarily taken to encompass the events. PREDICCS shielded SEP dose rates have been scaled to the location of MSL (1.12 AU, 1.27 AU, and 1.46 AU, respectively). We take note of the PREDICCS data gaps during DOY 31, DOY 60, and DOY 140. CRaTER measurements remain on the Moon's surface where half the sky is blocked by the Moon, hence CRaTER's background dose rate is lower than RAD by a factor of 2 in addition to the difference due to the distance from the Sun.

Using the indices from the power law fits, the shielded dose rates produced by PREDICCS are scaled to the location of MSL. For the January and March events, the dose rates are scaled from Earth to $1.12 \mathrm{AU}$ and $1.27 \mathrm{AU}$, respectively. For the May event, we scale the dose rates from Mars to 1.46 AU since MSL was magnetically well connected with Mars at this time (Posner et al. 2013).

Figure 5 shows dose rates for the January (Fig. 5a), March (Fig. 5b), and May (Fig. 5c) events. Included are PREDICCS shielded SEP dose rates scaled to the location of MSL, dose rates from MSL/RAD during its cruise, and CRaTER D1-D2 and microdosimeter dose rates at $1 \mathrm{AU}$. All dose rates have been hourly averaged. For data gaps, we linearly interpolate between the previous and next existing data point.

\subsection{Dose}

Dose is a quantity defined as the amount of energy deposited by radiation in a material per unit mass. The unit for dose is gray (Gy) where $1 \mathrm{~Gy}=1 \mathrm{~J} \mathrm{~kg}^{-1}$. The 30-day limits are based upon exposures to a reference radiation (gammas). Protons and other heavier charged particles can produce the same levels of damage as gammas, but at lower doses. Hence, in order to compare these doses to the 30-day limits, they must be scaled by their Relative Biological Effectiveness (RBE) for producing these acute radiation effects. These scaled doses, in units of Gray-equivalent (Gy-eq), are obtained using

$$
D(\mathrm{~Gy}-\mathrm{eq})=D(\mathrm{~Gy}) \times \mathrm{RBE},
$$

where we use the recommended RBE value of 1.5 for highenergy protons (NCRP 2000).

The GCR background measured by RAD and CRaTER includes particle events from heavy ions which have a different RBE than high-energy protons. We therefore must subtract out the GCR background to get an accurate description of the short-term radiation effects from SEP protons. Zeitlin et al. (2013) determined the GCR background dose rate measured by RAD's B detector (after converting to dose rate in water) to be $0.0481 \mathrm{cGy} /$ day $\pm 0.0080 \mathrm{cGy} /$ day. For CRaTER, Joyce et al. (2013) used the Badhwar-O'Neill model (O'Neill 2006) to approximate the GCR background dose rate as seen by CRaTER at the Moon for the January, March, and May 2012 solar events. The respective GCR background dose rates were $0.0213 \mathrm{cGy} / \mathrm{day}, 0.0203 \mathrm{cGy} / \mathrm{day}$, and 0.0199 cGy/day.

\subsection{Accumulated dose}

We calculate the total dose accumulated during the solar events by multiplying each hourly averaged dose rate by $1 \mathrm{~h}$, then 
(a)

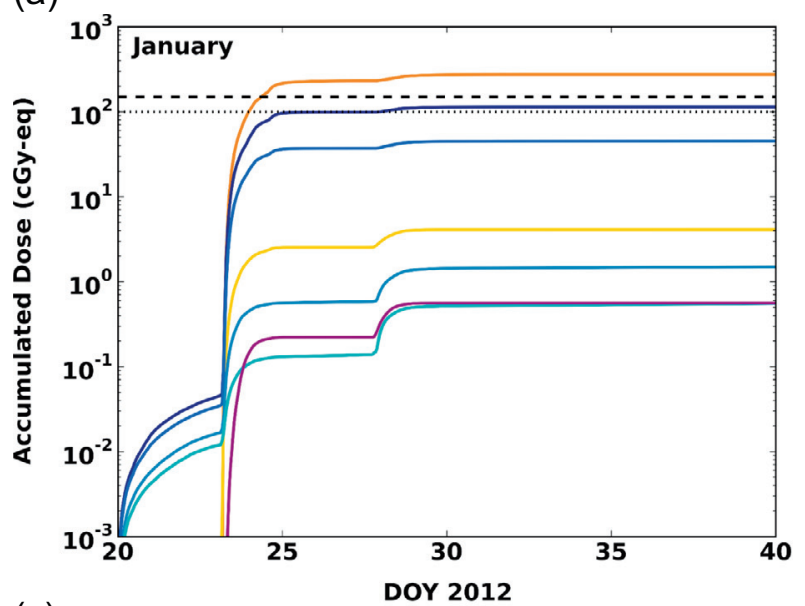

(c)

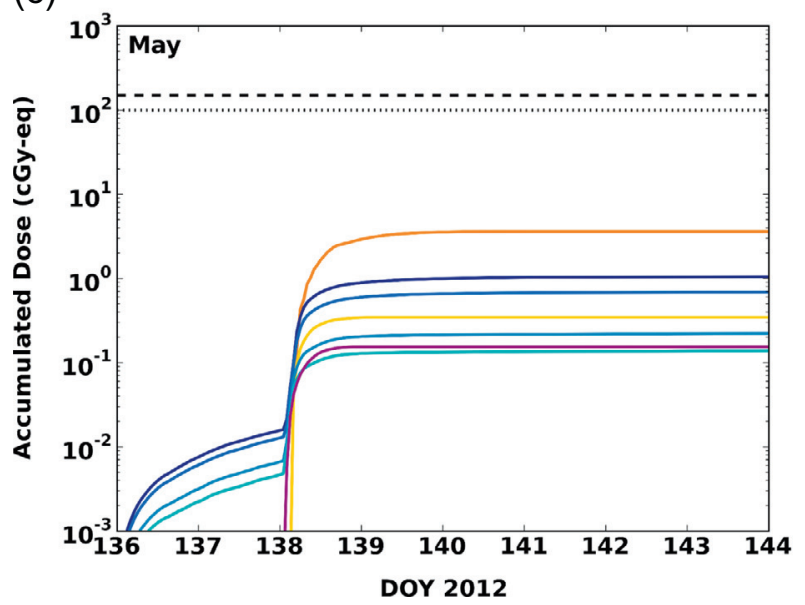

(b)
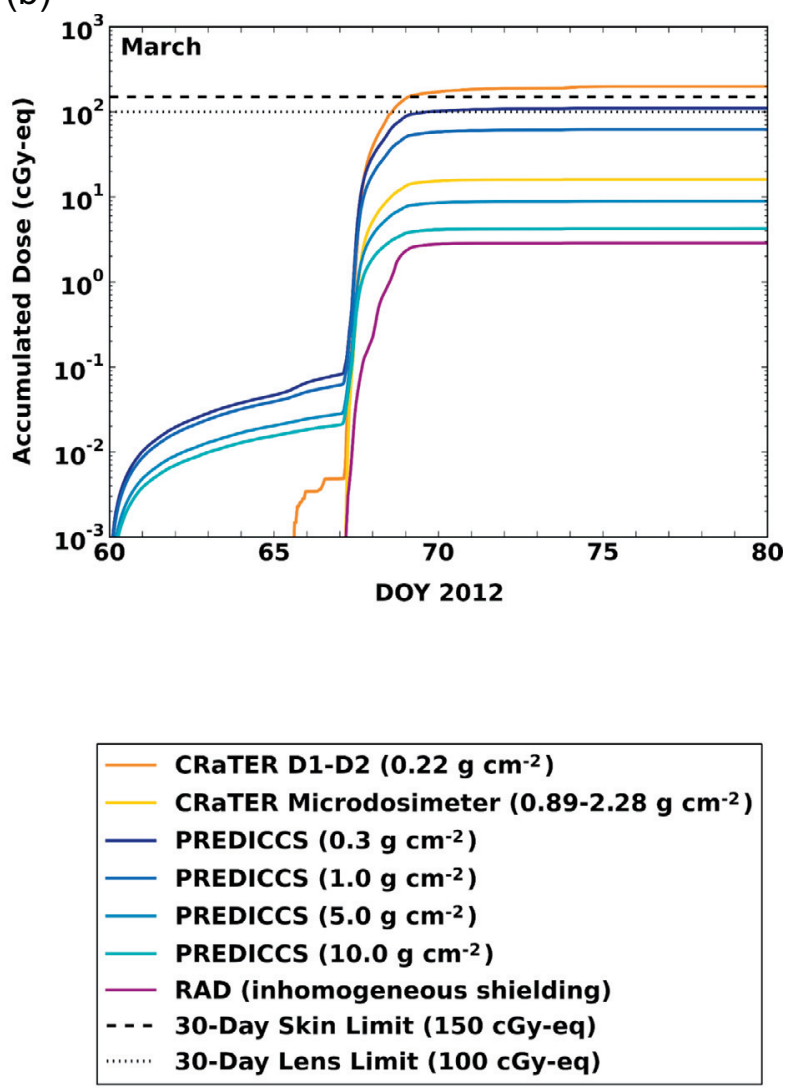

Fig. 6. Dose accumulated during the January (a), March (b), and May (c) 2012 solar events. The beginning and ending times are arbitarily taken to encompass the events. The dotted and dashed lines represent the 30-day limit for lens (100 cGy-eq) and skin (150 cGy-eq), respectively. The GCR background dose rate measured by CRaTER D1-D2, microdosimeter, and RAD has been subtracted out. The small increase in DOY 65 seen by CRaTER D1-D2 and PREDICCS (under $1.0 \mathrm{~g} \mathrm{~cm}^{-2}$ ) is from the flare that occurred prior to the March 7 event.

summing over all doses. The cumulative dose for the January event (Fig. 6a), March event (Fig. 6b), and May event (Fig. 6c) is shown in Figure 6. The NASA 30-day limits for skin (150 cGy-eq) and lens (100 cGy-eq) are displayed by the two dashed lines near the top of each plot.

The CRaTER dose exceeds the skin and lens limits during the January and March events while remaining well under the limits during the May event. PREDICCS $0.3 \mathrm{~g} \mathrm{~cm}^{-2}$ shielded dose reached as high as 114.13 cGy-eq during the January event - a factor of 1.14 above the lens limit. For the March event, this factor was about 1.10. The accumulated dose for each level of shielding is summarized in Table 1 in Section 5. Also included in the table is the dose accumulated in $10.0 \mathrm{~g} \mathrm{~cm}^{-2} \mathrm{H}_{2} \mathrm{O}$ - a proxy for blood-forming organs (BFOs), heart, and central nervous system (CNS). The 30-day limits are 25 cGy-eq, 25 cGy-eq, and 50 cGy-eq, respectively ( $\mathrm{Wu}$ et al. 2009). The greatest dose accumulated was during the March event, reaching as high as 3.72 cGy-eq for $0.3 \mathrm{~g} \mathrm{~cm}^{-2}$ aluminum shielding - a factor of almost 7 under the BFO and heart 30-day limit. We can therefore infer that the health risks to BFOs, the heart, and the CNS are minimal if astronauts experienced these events with minimal shielding.

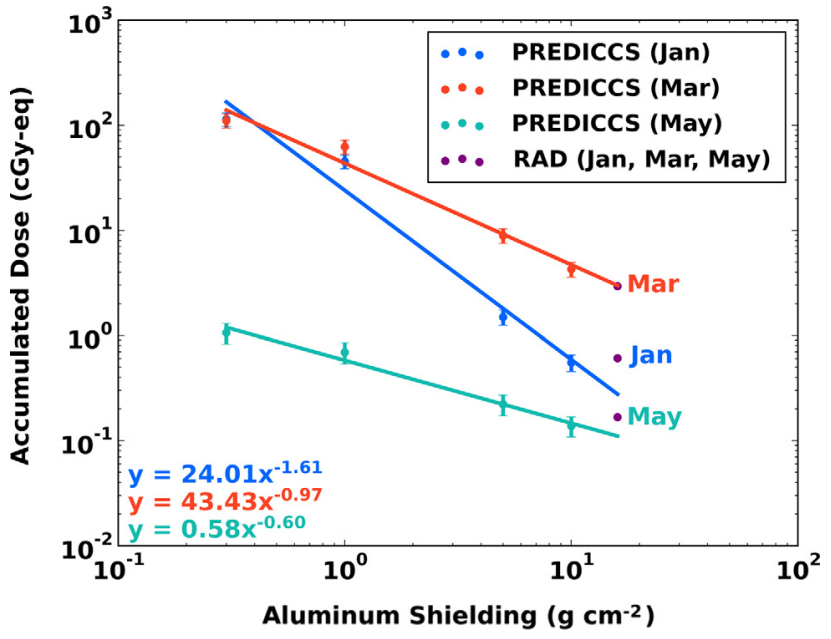

Fig. 7. PREDICCS accumulated dose as a function of aluminum shielding for the January events (Blue), March events (Red), and May event (Green). A power law (solid lines) is fit to the PREDICCS predictions. Dose accumulated by RAD (purple dots) is shown for each event (labeled at the right of each dot) at the average shielding of roughly $16 \mathrm{~g} \mathrm{~cm}^{-2}$. In the lower-left corner are the mathematical forms of the PREDICCS predictions. 
Table 2. Accumulated dose for RAD, PREDICCS at the location of MSL during each solar event, and CRaTER at the moon.

\begin{tabular}{lcccc}
\hline \hline & Al shielding $\left(\mathrm{g} \mathrm{cm}^{-2}\right)$ & January (cGy-eq) & March (cGy-eq) & May (cGy-eq) \\
\hline PREDICCS $\left(\mathrm{H}_{2} \mathrm{O}=1.0 \mathrm{~g} \mathrm{~cm}^{-2}\right)$ & 0.3 & $114.13 \pm 17.06$ & $110.30 \pm 17.11$ & $1.06 \pm 0.24$ \\
PREDICCS $\left(\mathrm{H}_{2} \mathrm{O}=1.0 \mathrm{~g} \mathrm{~cm}^{-2}\right)$ & 1.0 & $45.18 \pm 6.77$ & $62.27 \pm 9.59$ & $0.69 \pm 0.16$ \\
PREDICCS $\left(\mathrm{H}_{2} \mathrm{O}=1.0 \mathrm{~g} \mathrm{~cm}^{-2}\right)$ & 5.0 & $1.49 \pm 0.25$ & $8.89 \pm 1.43$ & $0.22 \pm 0.05$ \\
PREDICCS $\left(\mathrm{H}_{2} \mathrm{O}=1.0 \mathrm{~g} \mathrm{~cm}^{-2}\right)$ & 10.0 & $0.55 \pm 0.10$ & $4.26 \pm 0.68$ & $0.14 \pm 0.03$ \\
CRaTER D1-D2 & 0.22 & 275.79 & 199.74 & 3.80 \\
CRaTER Microdosimeter & $0.89 / 2.28$ & 4.36 & 16.41 & 0.42 \\
RAD & -16.0 & 0.61 & 2.94 & 0.17 \\
PREDICCS $/ \mathrm{RAD} \%$ Difference & - & $-54 \%$ & $+2 \%$ & $-38 \%$ \\
PREDICCS $\left(\mathrm{H}_{2} \mathrm{O}=10.0 \mathrm{~g} \mathrm{~cm}^{-2}\right)$ & 0.3 & $0.48 \pm 0.08$ & $3.72 \pm 0.60$ & $0.13 \pm 0.03$ \\
PREDICCS $\left(\mathrm{H}_{2} \mathrm{O}=10.0 \mathrm{~g} \mathrm{~cm}^{-2}\right)$ & 1.0 & $0.36 \pm 0.08$ & $3.55 \pm 0.57$ & $0.12 \pm 0.03$ \\
PREDICCS $\left(\mathrm{H}_{2} \mathrm{O}=10.0 \mathrm{~g} \mathrm{~cm}^{-2}\right)$ & 5.0 & $0.29 \pm 0.05$ & $2.81 \pm 0.45$ & $0.10 \pm 0.02$ \\
PREDICCS $\left(\mathrm{H}_{2} \mathrm{O}=10.0 \mathrm{~g} \mathrm{~cm}^{-2}\right)$ & 10.0 & & $2.23 \pm 0.36$ & $0.09 \pm 0.02$ \\
\hline
\end{tabular}

\section{Discussion}

\subsection{Validating PREDICCS}

To validate PREDICCS at distances beyond Earth, we compare the shielded accumulated dose of PREDICCS to that of RAD. Shown in Figure 7 is the accumulated dose of PREDICCS as a function of aluminum shielding for the January, March, and May events. Since the shielding surrounding RAD (roughly $16 \mathrm{~g} \mathrm{~cm}^{-2}$; Zeitlin et al. 2013) is beyond the highest shielding of PREDICCS $\left(10 \mathrm{~g} \mathrm{~cm}^{-2}\right)$, a power law is fit to the PREDICCS predictions. The mathematical form of each power law is given in the lower-left corner. RAD measured 0.61 cGy-eq, 2.94 cGy-eq, and 0.17 cGy-eq for the January, March, and May events, respectively. This corresponds to a percent difference between the PREDICCS power law and $\mathrm{RAD}$ of $-54 \%,+2 \%$, and $-38 \%$ for each event. The accumulated dose for PREDICCS, RAD, and CRaTER is summarized in Table 2 .

There is an overall underestimation of accumulated dose by PREDICCS when compared to RAD. This is also evident in Figure 5 where PREDICCS dose rates fall off quicker than CRaTER and RAD. The discrepancy between the $0.3 \mathrm{~g} \mathrm{~cm}^{-2}$ shielded dose rate of PREDICCS and dose rates from CRaTER $\left(\sim 0.22 \mathrm{~g} \mathrm{~cm}^{-2}\right.$ aluminum-equivalent shielding) was concluded to be due to EMMREM not handling low-energy protons well (Joyce et al. 2013). Heavy ions, not included in PREDICCS, may still account for increased dose from fragmentation within the heavier shielding.

\subsection{Reduction from shielding}

Shielding mitigates the dose accumulated by astronauts from SEPs as shown in Figure 7. The percent each level of shielding reduces the accumulated dose with respect to $0.3 \mathrm{~g} \mathrm{~cm}^{-2}$ aluminum shielding is given in Table 3 for each solar event. For $1.0 \mathrm{~g} \mathrm{~cm}^{-1}$ shielding, the dose accumulated during the January and March events is further reduced by roughly half $(\sim 40-60 \%)$. For 5.0 and $10.0 \mathrm{~g} \mathrm{~cm}^{-1}$ shielding, the dose is almost entirely mitigated ( $>90 \%)$.

The effectiveness of shielding for the May event is considerably lower than for the January and March events. For $1.0 \mathrm{~g} \mathrm{~cm}^{-2}$ shielding, the dose reduction for the May event only reaches $35 \%$. For shielding of $5.0 \mathrm{~g} \mathrm{~cm}^{-2}$ and above, the dose reduction for the January and March events is greater than
Table 3. Percent PREDICCS accumulated dose reduced by shielding for each solar event.

\begin{tabular}{lccc}
\hline & $\begin{array}{c}\text { January } \\
(\%)\end{array}$ & $\begin{array}{c}\text { March } \\
(\%)\end{array}$ & $\begin{array}{c}\text { May } \\
(\%)\end{array}$ \\
\hline $\begin{array}{l}\text { Al shielding, } \mathrm{H}_{2} \mathrm{O}=1.0 \\
\left(\mathrm{~g} \mathrm{~cm}^{-2}\right)\end{array}$ & & & \\
0.3 & - & - & - \\
1.0 & 60.4 & 43.5 & 34.9 \\
5.0 & 98.7 & 91.9 & 79.2 \\
10.0 & 99.5 & 96.1 & 86.8 \\
$\mathrm{Al}$ shielding, $\mathrm{H}_{2} \mathrm{O}=10.0$ & & & \\
$\left(\mathrm{~g} \mathrm{~cm}^{-2}\right)$ & & & \\
0.3 & - & - & - \\
1.0 & 4.2 & 4.6 & 7.7 \\
5.0 & 25.0 & 24.5 & 23.1 \\
10.0 & 39.6 & 40.1 & 30.8 \\
\hline
\end{tabular}

Notes. Each percent is with respect to $0.3 \mathrm{~g} \mathrm{~cm}^{-2}$ aluminum shielding.

$90 \%$. However for the May event, the reduction is only $80-90 \%$. Referring to Figure 1, the peak intensities for lower energy protons measured by GOES-13/EPS/HEPAD for the May event are 1-2 orders of magnitude lower than the January and March events. However, the energy spectrum of the May event is much harder than the other events. Since shielding is less effective against higher energy protons, we see less of a benefit from higher levels of shielding for the May event than we do for the January and March events.

Also given in Table 3 are doses in $10.0 \mathrm{~g} \mathrm{~cm}^{-2} \mathrm{H}_{2} \mathrm{O}-\mathrm{a}$ proxy for BFOs, heart, and CNS. Beyond $0.3 \mathrm{~g} \mathrm{~cm}^{-2}$ aluminum shielding, the largest reduction is seen for the dose accumulated during the January and March events ( 40\%). The May event only saw a reduction of about $31 \%$ for $10 \mathrm{~g} \mathrm{~cm}^{-2}$ shielding.

\subsection{Radial dependence of accumulated dose}

Shown in Figure 8 is the accumulated dose along the interplanetary magnetic field as a function of heliocentric distance for the January, March, and May events. The indices, summarized in Table 4, are comparable to those of the Halloween 2003 event (Kozarev et al. 2010). Close to 1 AU, the dose accumulated during the January event exceeds the 30-day lens limit by 
P.R. Quinn et al.: Shielding in SEP environment using PREDICCS

(a)

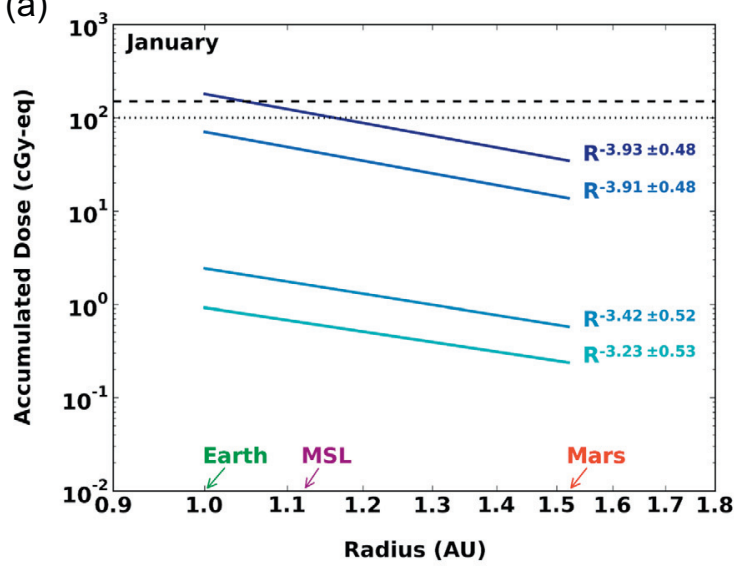

(c)

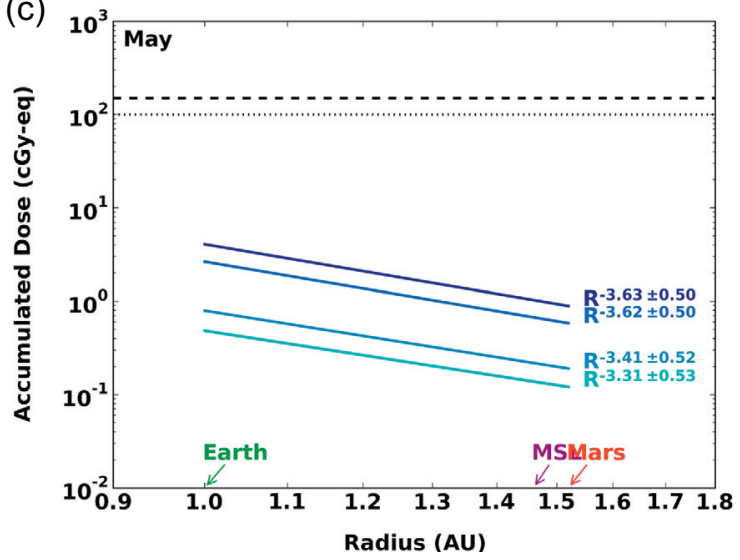

(b)

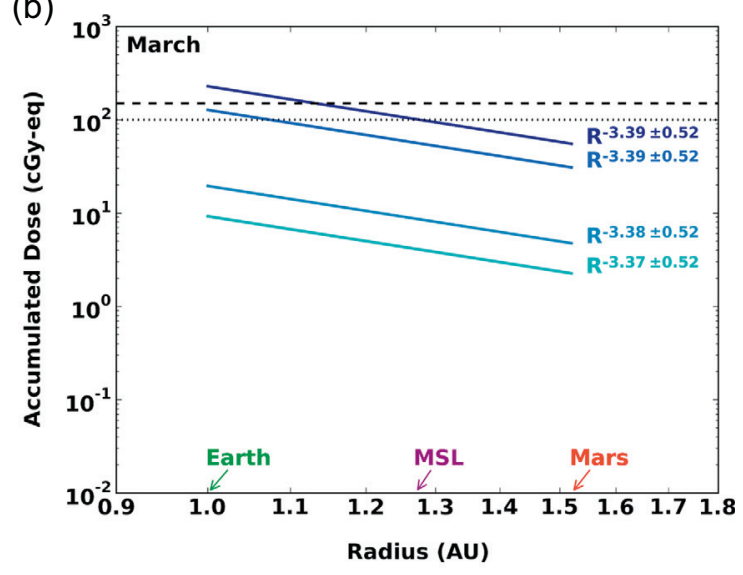

Fig. 8. PREDICCS accumulated dose along the interplanetary magnetic field between Earth and Mars as a function of heliocentric distance. The location of Earth, MSL, and Mars is shown at the bottom.

Table 4. Radial dependence of dose rate and accumulated dose between Earth and Mars.

\begin{tabular}{lcccc}
\hline \hline & \multicolumn{4}{c}{ Power indices for dose rate } \\
\cline { 2 - 5 } & January & March & May & Halloween 2003* \\
\hline Al shielding, $\mathrm{H}_{2} \mathrm{O}=1.0\left(\mathrm{~g} \mathrm{~cm}^{-2}\right)$ & $-4.27 \pm 0.47$ & $-3.15 \pm 0.54$ & $-4.34 \pm 0.46$ & $-4.57 \pm 0.45$ \\
0.3 & $-4.15 \pm 0.47$ & $-3.10 \pm 0.55$ & $-4.34 \pm 0.46$ & $-4.24 \pm 0.41$ \\
1.0 & $-4.24 \pm 0.47$ & $-3.40 \pm 0.52$ & $-3.80 \pm 0.49$ & $-4.26 \pm 0.41$ \\
5.0 & $-4.35 \pm 0.46$ & $-3.35 \pm 0.52$ & $-3.28 \pm 0.53$ & $-4.20 \pm 0.42$ \\
10.0 & & Power indices for accumulated dose & $-3.78 \pm 0.31$ \\
Al shielding, $\mathrm{H}_{2} \mathrm{O}=1.0\left(\mathrm{~g} \mathrm{~cm}^{-2}\right)$ & $-3.93 \pm 0.48$ & $-3.39 \pm 0.52$ & $-3.63 \pm 0.50$ & $-3.59 \pm 0.27$ \\
0.3 & $-3.91 \pm 0.48$ & $-3.39 \pm 0.52$ & $-3.62 \pm 0.50$ & $-3.59 \pm 0.27$ \\
1.0 & $-3.42 \pm 0.52$ & $-3.38 \pm 0.52$ & $-3.41 \pm 0.52$ & $-3.60 \pm 0.27$ \\
5.0 & $-3.23 \pm 0.53$ & $-3.37 \pm 0.52$ & $-3.31 \pm 0.53$ & \\
10.0 & & & &
\end{tabular}

* Kozarev et al. (2010).

a factor of 1.8 for $0.3 \mathrm{~g} \mathrm{~cm}^{-2}$ shielding and a factor of 0.7 under the lens limit for $1.0 \mathrm{~g} \mathrm{~cm}^{-2}$ shielding. For the March event, $0.3 \mathrm{~g} \mathrm{~cm}^{-2}$ and $1.0 \mathrm{~g} \mathrm{~cm}^{-2}$ exceed the lens limit by factors of 2.3 and 1.3, respectively. The May event shows the least concern of the three events at $1 \mathrm{AU}$ with accumulated dose of approximately $4.1 \mathrm{cGy-eq}$ and below for all shielding.

In interplanetary space near Mars (approximately 1.5 AU during this time), the dose accumulated during the January, March, and May events is approximately 34 cGy-eq, 55 cGyeq, and $1 \mathrm{cGy}$-eq, respectively, for $0.3 \mathrm{~g} \mathrm{~cm}^{-2}$ aluminumequivalent shielding. Separately, the dose from these five specific events does not exceed the lens or skin limits. However, there are other major factors to consider. First, there are much stronger SEP events than the ones selected for this study. The dose accumulated at $1 \mathrm{AU}$ during the Halloween 2003 event reached as high as $1000 \mathrm{cGy}$-eq for aluminum-equivalent shielding of $1.0 \mathrm{~g} \mathrm{~cm}^{-2}$ (Kozarev et al. 2010). Second, the 30-day limit for lens and skin can easily be exceeded if more than one SEP event occurs within approximately 30 days of the next. Lastly, the SEP events in 2012 only contributed roughly $5 \%$ of the total accumulated dose during MSL's cruise to Mars (Zeitlin et al. 2013). The remaining dose is from GCRs. 
The radial dependence of accumulated dose observed by Dayeh 2010 exhibited a broken power law. The reason no break was observed in this study is because Dayeh et al. (2010) observed dose out to nearly 5 AU with the break occurring at approximately 3 AU. PREDICCS, however, only calculates dose rates at Earth and Mars. Within this range, the transport of particles along the magnetic field has a prominent radial component. With greater distance, the angle of the Parker spiral becomes mainly azimuthal, and radial particle transport depends on the less effective diffusion across the magnetic field.

\section{Summary and conclusion}

We have utilized PREDICCS to study how shielding reduces the short-term health risks for human space exploration missions within the Earth-Moon-Mars SEP radiation environment. We compared shielded dose rates and accumulated dose from PREDICCS to LRO/CRaTER at the Moon and MSL/RAD in its cruise phase from Earth to Mars during the January, March, and May 2012 solar events. During the cruise, MSL was magnetically well connected with Earth for the first phase and Mars during the latter phase.

We expressed the dose rate and accumulated dose along the interplanetary field as a function of heliocentric radius. The power law indices of these radial gradients are comparable to the Halloween 2003 event, given in Table 3. Near $1 \mathrm{AU}$, the dose accumulated during the January and March events exceeds the 30-day lens limit for low levels of shielding. In interplanetary space near Mars, neither the 30-day lens or skin limits are exceeded by these events. However, multiple events in a short time period or stronger events such as the Halloween 2003 event may easily exceed these limits.

The accumulated dose predicted by PREDICCS and measured by RAD is in reasonable agreement. PREDICCS underestimated the dose by approximately $54 \%$ and $38 \%$ for the January and May events, respectively. For the March event, PREDICCS and RAD were in remarkable agreement with PREDICCS only overestimating the dose by about $2 \%$. Thus, this study shows that PREDICCS is a valuable tool for characterizing the Earth-Moon-Mars SEP radiation environment and demonstrates how shielding mitigates the dose accumulated by astronauts.

Acknowledgements. Support for this work was provided by the NASA Lunar Reconnaissance Orbiter Project (NASA Contract NNG11PA03C), as well as various NASA grants (EMMREM, Grant NNX07AC14G; C-SWEPA, Grant NNX07AC14G; DoSEN, Grant NNX13AC89G; DREAM, Grant NNX10AB17A; and DREAM2; Grant NNX14AG13A) and an NSF grant (Sun-2-Ice, Grant AGS1135432). We thank the International Space Science Institute for supporting the Research Team: Radiation Interactions at Planetary Bodies (http://www.issibern.ch/teams/interactplanetbody/).

RAD is supported by NASA (HEOMD) under JPL Subcontract \#1273039 to Southwest Research Institute and in Germany by DLR and DLR's Space Administration Grant Numbers 50QM0501 and 50QM1201 to the Christian Albrechts University, Kiel. Part of this research was carried out at the Jet Propulsion Laboratory, California Institute of Technology, under a contract with the National Aeronautics and Space Administration. The data used in this paper may be retrieved from the NASA Planetary Data System

The GOES 8-15 X-ray/magnetic field/particle data are produced in real time by the NOAA Space Weather Prediction Center (SWPC) and are distributed by the NOAA National Geophysical Data Center (NGDC).

We would like to thank the reviewers and editor for their informative feedback, and Cary Zeitlin for the helpful discussions.

The editor thanks two anonymous referees for their assistance in evaluating this paper.

\section{References}

Anno, G.H., S.J. Baum, H.R. Withers, R.W. Young, R.W. Young, and R.W. Young. Symptomatology of acute radiation effects in humans after exposure to doses of 0.5-30 Gy. Health Phys., 56, 821-838, 1989.

Chin, G.S. Brylow, M. Foote, J. Garvin, J. Kasper, et al. Lunar reconnaissance orbiter overview: the instrument suite and mission. Space Sci. Rev., 129, 391-419, 2007, DOI: $10.1007 / \mathrm{s} 11214-007-9153-y$.

Cucinotta, F.A., M. Alp, B. Rowedder, and M.-H.Y. Kim. Safe days in space with acceptable uncertainty from space radiation exposure. Life Sci. Space. Res., 5, 31-38, 2015, DOI: $10.1016 /$ j.lssr.2015.04.002.

Cucinotta, F.A., S. Hu, N.A. Schwadron, K. Kozarev, L.W. Townsend, and M.-H.Y. Kim. Space radiation risk limits and earth-moon-mars environmental models. Space Weather, 8, S00E09, 2010, DOI: 10.1029/2010SW000572.

Dayeh, M.A., M.I. Desai, K. Kozarev, N.A. Schwadron, L.W. Townsend, M. PourArsalan, C. Zeitlin, and R.B. Hatcher. Modeling proton intensity gradients and radiation dose equivalents in the inner heliosphere using EMMREM: May 2003 solar events. Space Weather, 8, S00E07, 2010, DOI: $10.1029 / 2009$ SW000566.

Ding, L.-G., Y. Jiang, and G. Li. Are there two distinct solar energetic particle releases in the 2012 May 17 ground level enhancement event? Astrophys. J., 818, 169, 2016, DOI: $10.3847 / 0004-637 \mathrm{X} / 818 / 2 / 169$.

Gopalswamy, N., P. Mäkelä, S. Yashiro, H. Xie, S. Aökiyama, and N. Thakur. High-energy solar particle events in cycle 24. J. Phys. Conf. Ser., 642, 012012, 2015, DOI: $10.1088 / 1742-6596 / 642 / 1 / 012012$.

Grotzinger, J.P., J. Crisp, A.R. Vasavada, R.C. Anderson, C.J. Baker, et al. Mars science laboratory mission and science investigation. Space Sci. Rev., 170, 5-56, 2012, DOI: $10.1007 / \mathrm{s} 11214-012-9892-2$

Hassler, D.M., C. Zeitlin, R.F. Wimmer-Schweingruber, S. Bottcher, C. Martin, et al. The radiation assessment detector (RAD) investigation. Space Sci. Rev., 170, 503-558, 2012, DOI: $10.1007 / \mathrm{s} 11214-012-9913-1$

Jokipii, J.R., and E.H. Levy. Effects of particle drifts on the solar modulation of galactic cosmic rays. Astrophys. J., 213, L85-L88, 1977, DOI: $10.1086 / 182415$.

Joyce, C.J., N.A. Schwadron, J.K. Wilson, H.E. Spence, J.C. Kasper, et al. Validation of PREDICCS using LRO/CRaTER observations during three major solar events in 2012. Space Weather, 11(6), 350-360, 2013, DOI: 10.1002/swe.20059.

Kozarev, K., N.A. Schwadron, M.A. Dayeh, L.W. Townsend, M.I. Desai, and M. PourArsalan. Modeling the 2003 Halloween events with EMMREM: energetic particles, radial gradients and coupling to MHD. Space Weather, 8, S00E08, 2010, DOI: 10.1029/2009SW000550.

Lee, M.A., and L.A. Fisk. The role of particle drifts in solar modulation. Astrophys. J., 248, 836-844, 1981, DOI: $10.1086 / 159208$.

Li, C., K.A. Firoz, L.P. Sun, and L.I. Miroshnichenko. Electron and proton acceleration during the first ground level enhancement event of solar cycle 24. Astrophys. J., 770, 34, 2013, DOI: $10.1088 / 0004-637 \mathrm{X} / 770 / 1 / 34$

Liu, Y.D., J.G. Luhmann, N. Lugaz, C. Möstl, J.A. Davies, S.D. Bale, and R.P. Lin. On sun-to-earth propagation of coronal mass ejections. Astrophys. J., 769, 45, 2013, DOI: $10.1088 / 0004-637 \mathrm{X} / 769 / 1 / 45$. 
P.R. Quinn et al.: Shielding in SEP environment using PREDICCS

Looper, M.D., J.E. Mazur, J.B. Blake, H.E. Spence, N.A. Schwadron, M.J. Golightly, A.W. Case, J.C. Kasper, and L.W. Townsend. The radiation environment near the lunar surface: CRaTER observations and Geant4 simulations. Space Weather, 11(4), 142-152, 2013, DOI: 10.1002/swe.20034.

Mazur, J.E., W.R. Crain, M.D. Looper, D.J. Mabry, J.B. Blake, A.W. Case, M.J. Golightly, J.C. Kasper, and H.E. Spence. New measurements of total ionizing dose in the lunar environment. Space Weather, 9, S07002, 2011, DOI: 10.1029/2010SW000641.

NCRP. Guidance on radiation received in space activities. NCRP Report 98, 1989.

NCRP. National council on radiation protection and measurements, recommendations of dose limits for low earth orbit. NCRP Report 132, 2000.

NRC. Radiobiological factors in manned spaceflight. In: W.H. Langham, Editor, Report of Space Radiation Study Panel of the Life Sciences Committee, National Academy Press, Washington, DC, 1967.

NRC. Radiation protection guides and constraints for space-mission and vehicle-design studies involving nuclear systems. In: W.H. Langham, D. Grahn, Editors, Report of the Radiobiologic Advisory Panel of the Committee on Space Medicine Space Science Board, National Academy Press, Washington, DC, 1970.

O’Neill, P.M.. Badhwar O'Neill galactic cosmic ray model update based on advanced composition explorer (ACE) energy spectra from 1997 to present. Adv. Space Res., 37, 1727-1733, 2006, DOI: $10.1016 /$ j.asr.2005.02.001.

Posner, A., D. Odstrĉil, P. MacNeice, P. MacNeice, L. Rastaetter, C. Zeitlin, et al. The Hohmann-Parker effect measured by the Mars science laboratory on the transfer from earth to mars: consequences and opportunities. Planet. Space Sci., 89, 127-139, 2013, DOI: 10.1016/j.pss.2013.09.013.

Quinn, P.R., N.A. Schwadron, and E. Möbius. Transport of helium pickup ions within the focusing cone: reconciling STEREO observations with IBEX. Astrophys. J., 824, 142, 2016, DOI: $10.3847 / 0004-637 \mathrm{X} / 824 / 2 / 142$.

Ruffolo, D. Effect of adiabatic deceleration on the focused transport of solar cosmic rays. Astrophys. J., 442, 861-874, 1995, DOI: $10.1086 / 175489$.

Schwadron, N.A., T. Baker, B. Blake, A.W. Case, J.F. Cooper, et al. Lunar radiation environment and space weathering from the cosmic ray telescope for the effects of radiation (CRaTER). J. Geophys. Res. [Planets], 117, E00H13, 2012, DOI: 10.1029/2011JE003978.

Schwadron, N.A., L. Townsend, K. Kozarev, M.A. Dayeh, and F. Cucinotta. Earth-moon-mars radiation environment module framework. Space Weather, 8, S00E02, 2010, DOI: $10.1029 / 2009$ SW000523.

Skilling, J. Cosmic rays in the galaxy: convection or diffusion? Astrophys. J., 170, 265, 1971, DOI: 10.1086/151210.

Spence, H.E., A.W. Case, M.J. Golightly, T. Heine, B.A. Larsen, et al. CRaTER: the cosmic ray telescope for the effects of radiation experiment on the lunar reconnaissance orbiter mission. Space Sci. Rev., 150, 243-284, 2010, DOI: $10.1007 / \mathrm{s} 11214-009-9584-8$.

Wilson, J.W., L.W. Townsend, J.E. Nealy, S.Y. Chun, B.S. Hong, W.W. Buck, S.L. Lamkin, B.D. Ganapol, F. Khan, and F.A. Cucinotta. BRYNTRN: a baryon transport model. NASA STI/Recon Technical Report No. 89, 1989.

Wu, H., J.L. Huff, R. Casey, M.-H. Kim, and F.A. Cucinotta. Risk of acute radiation syndromes due to solar particle events, 2009, NASA Johnson Space Center, Houston, HRP-47052.

Zeitlin, C., D.M. Hassler, F.A. Cucinotta, B. Ehresmann, R.F. Wimmer-Schweingruber, et al. Measurements of energetic particle radiation in transit to mars on the mars science laboratory. Science, 340, 1080-1084, 2013, DOI: $10.1126 /$ science.1235989.

Cite this article as: Quinn PR, Schwadron NA, Townsend LW, Wimmer-Schweingruber RF, Case AW, et al. Modeling the effectiveness of shielding in the earth-moon-mars radiation environment using PREDICCS: five solar events in 2012. J. Space Weather Space Clim., 7, A16, 2017, DOI: $10.1051 / \mathrm{swsc} / 2017014$.

\section{Appendix A}

\section{Notation}

\begin{tabular}{ll} 
ARS & Acute Radiation Syndrome \\
AU & Astronomical Unit \\
BFOs & Blood-Forming Organs \\
BRYNTRN & BaRYN TRaNsport model \\
CME & Coronal Mass Ejection \\
CNS & Central Nervous System \\
COSTEP & COmprehensive SupraThermal and Energetic Particle Analyzer \\
CRaTER & Cosmic Ray Telescope for the Effects of Radiation \\
DOY & Day of Year \\
EMMREM & Earth-Moon-Mars Radiation Environment Module \\
EPREM & Energetic Particle Radiation Environment Module \\
EPS & Energetic Particle Sensors \\
GCR & Galactic Cosmic Ray \\
GOES-13 & Geostationary Operational Environment Satellite-13 \\
HEPAD & High Energy Proton and Alpha Detector \\
LRO & Lunar Reconnaissance Orbiter \\
MSL & Mars Science Laboratory \\
PEL & Permissible Exposure Limit \\
PREDICCS & Predictions of radiation from REleASE, EMMREM, and Data Incorporating the CRaTER, \\
RAD & COSTEP, and other SEP measurements \\
RBE & Radiation Assessment Detector \\
REleASE & Relative Biological Effectiveness \\
SEP & Relativistic Electron Alert System for Exploration \\
\hline
\end{tabular}

